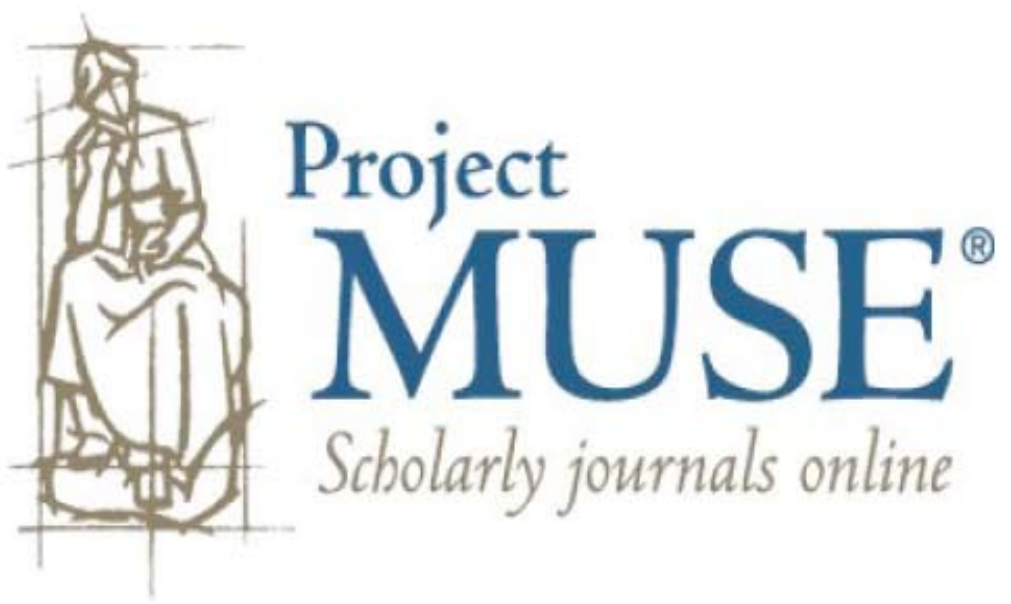




\title{
GALOIS STRUCTURE OF ZARISKI COHOMOLOGY FOR WEAKLY RAMIFIED COVERS OF CURVES
}

\author{
By Bernhard Köck
}

\begin{abstract}
We compute equivariant Euler characteristics of locally free sheaves on curves, thereby generalizing several results of Kani and Nakajima. For instance, we extend Kani's computation of the Galois module structure of the space of global meromorphic differentials which are logarithmic along the ramification locus from the tamely ramified to the weakly ramified case.
\end{abstract}

Introduction. Let $X$ be a smooth projective curve defined over an algebraically closed field $k$ of characteristic $p$, and let $G \subseteq \operatorname{Aut}(X / k)$ be a finite subgroup of automorphisms of $X$. The goal of this paper is to compute the Galois module structure of the Zariski cohomology groups of $X$ with values in a $G$-equivariant locally free sheaf $\mathcal{E}$ on $X$ such as the ideal sheaf of a $G$-stable finite subset of points on $X$ or the sheaf of differentials on $X$.

Our first result, see Theorem 3.1, is an explicit formula for the equivariant Euler characteristic

$$
\chi(G, X, \mathcal{E}):=\left[H^{0}(X, \mathcal{E})\right]-\left[H^{1}(X, \mathcal{E})\right]
$$

considered as an element of the Grothendieck group $K_{0}(G, k)$ of all finitely generated modules over the group ring $k[G]$. It describes $\chi(G, X, \mathcal{E})$ in terms of the rank and degree of $\mathcal{E}$, the genus $g_{Y}$ of the quotient curve $Y:=X / G$, the order of $G$ and of the higher ramification groups $G_{P, s}, P \in X, s \geq 0$, and the representations of the decomposition group $G_{P}$ on the fibre $\mathcal{E}(P)$ and on the cotangent space $\mathfrak{m}_{P} / \mathfrak{m}_{P}^{2}$ for $P \in X$.

In the case the cover $\pi: X \rightarrow Y$ is tamely ramified, this formula becomes Theorem 1.1 in [Kö2], see Remark 3.2. In particular, if the order of $G$ is not divisible by $p$, it implies the main result of the paper [EL] by Ellingsrud and Lønsted, which in turn generalizes the classical Chevalley-Weil formula, see [Kö2]. While Theorem 3.1 of the present paper has the advantage of being available in general, i.e. without any assumption on the ramification of $\pi$ or on the group $G$, it has the disadvantage of computing the equivariant Euler characteristic only in the "weak" Grothendieck group $K_{0}(G, k)$, i.e. it yields only composition factors. In particular, if $p$ divides the order of $G$, we need further input to describe the actual

Manuscript received November 21, 2002; revised June 16, 2003.

American Journal of Mathematics 126 (2004), 1085-1107. 
$k[G]$-isomorphism class of the cohomology groups $H^{0}(X, \mathcal{E})$ and $H^{1}(X, \mathcal{E})$, even if one of them vanishes.

Such an input is provided by Theorem 1.1, parts of which may already be found in the literature, see Remark 1.2. It gives a criterion for any fractional ideal in a local Galois extension to have a normal basis element. We now describe the main application of this criterion in more detail in the geometric context considered so far. We say (as in Erez' paper [Er1]) that the cover $\pi$ is weakly ramified, if all second ramification groups $G_{P, 2}, P \in X$, are trivial, i.e., if both tame and the simplest kind of wild ramification are allowed. This condition is less special than it appears; in fact it is generically satisfied because, for any ordinary (i.e., $p$-rank $\left.g_{X}\right)$ curve $X$ and any arbitrary finite subgroup $G$ of $\operatorname{Aut}(X / k)$, the cover $\pi: X \rightarrow X / G$ is weakly ramified by the Deuring-Shafarevic formula, see Theorem 1.2 on p. 4 in [Pi]. The main application of the criterion mentioned above is the following fact, see the proof of Theorem 2.1(a): Let $\pi$ be weakly ramified and let $D=\sum_{P \in X} n_{P}[P]$ be a $G$-equivariant divisor on $X$ such that $n_{P} \equiv-1 \bmod e_{P}^{\mathrm{W}}$ for all $P \in X$ (where $e_{P}^{\mathrm{W}}$ denotes the order of the (first) ramification group $G_{P, 1}$ ); then the direct image $\pi_{*}\left(\mathcal{O}_{X}(D)\right)$ of the associated $G$-equivariant invertible sheaf $\mathcal{O}_{X}(D)$ is a locally free $\mathcal{O}_{Y}[G]$-module. Using a standard argument in geometric Galois module theory (see Chinburg's paper [Ch] for the version most suitable for our purposes), we obtain from this fact that the equivariant Euler characteristic $\chi\left(G, X, \mathcal{O}_{X}(D)\right)$ lies in the image of the (injective) Cartan homomorphism $K_{0}(k[G]) \rightarrow K_{0}(G, k)$ and, moreover, that $H^{0}\left(X, \mathcal{O}_{X}(D)\right)$ and $H^{1}\left(X, \mathcal{O}_{X}(D)\right)$ are projective $k[G]$-modules, if one of them vanishes, see Theorem 2.1(a).

Applying this observation to the divisor $E:=\sum_{P \in X}\left(e_{P}^{\mathrm{W}}-1\right)[P]$ and the abovementioned "weak" formula for $\chi(G, X, \mathcal{E})$ to the invertible $G$-module $\mathcal{E}=\mathcal{O}_{X}(E)$ we construct in Theorem 4.3 a certain canonical projective $k[G]$-module $N_{G, X}$ (depending only on the action of $G$ on $X$ ) which embodies a global relation between the local data $\mathfrak{m}_{P} / \mathfrak{m}_{P}^{2}, P \in X$; here, the cotangent space $\mathfrak{m}_{P} / \mathfrak{m}_{P}^{2}$ of $X$ at $P$ should be considered as a one-dimensional representation of the decomposition group $G_{P}$. Moreover, using our "weak" formula again, we express $\chi\left(G, X, \mathcal{O}_{X}(D)\right)$ as an integral linear combination of classes of explicit projective $k[G]$-modules, one of which is $N_{G, X}$, see Theorem 4.5. These theorems generalize results of Kani and Nakajima from the tamely ramified to the weakly ramified case, see Remarks 4.4 and 4.6. Our approach to these theorems (described above) generalizes the one used in [Kö2] (a special case of which may already be found in Borne's thesis [Bo1]), but it is quite different from the ones used by Kani and Nakajima.

Finally we give the following two applications of these theorems. First, we compute the $k[G]$-module structure of the first cohomology group $H^{1}(X, \mathcal{I}(S))$ of $X$ with values in the ideal sheaf $\mathcal{I}(S)$ of any $G$-stable finite subset $S$ of $X$ which contains all wildly ramified points, see Corollary 4.7. We refer the reader to Pink's paper [Pi] for the significance of this ideal sheaf in his proof of a $p$-adic Grothendieck-Ogg-Shafarevic formula. Secondly, if $S$ contains not only all wildly ramified but all ramified points, we prove that the direct sum of the $k[G]$-module 
$N_{G, X}$ with the space $H^{0}\left(X, \Omega_{X}(S)\right)$ of global meromorphic differentials, which are logarithmic along $S$, is a free $k[G]$-module, see Corollary 4.8. This result generalizes Theorem 2 in Kani's paper [Ka] again from the tamely ramified to the weakly ramified case. In the tamely ramified case, Kani furthermore deduces the $k[G]$-isomorphism class of the space $H^{0}\left(X, \Omega_{X}\right)$ of all global holomorphic differentials from this result, see Theorem 3 in $[\mathrm{Ka}]$. It would be interesting to know whether this can also be done in the weakly ramified case.

At this point we moreover mention that the conditions $\pi$ is weakly ramified and $n_{P} \equiv-1 \bmod e_{P}^{\mathrm{w}}$ for all $P \in X$ are not only sufficient, but also necessary for $\pi_{*}\left(\mathcal{O}_{X}(D)\right)$ to be locally free over $\mathcal{O}_{Y}[G]$, see Theorem 1.1 , and, if the degree of $D$ is sufficiently large, also for $H^{0}\left(X, \mathcal{O}_{X}(D)\right)$ to be a projective $k[G]$-module, see Theorem 2.1(b). Without the assumption on the degree of $D$, it might be true that these conditions are necessary for $R \Gamma\left(X, \mathcal{O}_{X}(D)\right)$ to be quasi-isomorphic to a perfect complex of $k[G]$-modules (see Question 2.2).

The reader may also wish to consult the paper [Vi] by Vinatier for the current state of the art in the Galois module theory of weakly ramified extensions of number fields.

Acknowledgments. I would like to thank Niels Borne, Boas Erez, Ian Leary, Richard Pink and Vic Snaith for helpful discussions and for their encouraging interest. In particular, Niels Borne has drawn my attention to Nakajima's paper [Na3] which is crucial for Theorem 2.1(b); Ian Leary has helped me with the important Lemma 4.2; and Vic Snaith has provided a first approach to a central part of Theorem 1.1. Finally, I would like to thank the referee for carefully reading the paper and for giving many helpful comments improving its presentation.

1. The normal basis theorem for fractional ideals in local Galois extensions. In this section we explicitly describe those fractional ideals in any finite Galois extension of local fields for which the normal basis theorem holds, i.e., which are free (of rank 1) over the corresponding group ring.

Let $L / K$ be a finite Galois extension of local fields with Galois group $G$ and with residue fields of (positive) characteristic $p$. The corresponding extensions of discrete valuation rings, of maximal ideals and of (perfect) residue fields are denoted by $\mathcal{O}_{L} / \mathcal{O}_{K}, \mathfrak{m}_{L} / \mathfrak{m}_{K}$ and $\lambda / \kappa$, respectively. For any integer $s \geq-1$, let $G_{s}$ denote the sth ramification group of the extension $L / K$. We recall (see Chapitre IV in [Se1]): The ramification groups form a chain

$$
G=G_{-1} \supseteq G_{0} \supseteq G_{1} \supseteq G_{2} \supseteq \cdots
$$

of normal subgroups of $G ; G_{0}=\operatorname{ker}(G \rightarrow \operatorname{Gal}(\lambda / \kappa))$ is the inertia subgroup; $G_{0} / G_{1}$ is a cyclic group of order prime to $p ; G_{s} / G_{s+1}$ is an abelian group of exponent $p$ for $s \geq 1 ; G_{s}$ is the trivial group for $s$ sufficiently big. The extension 
$L / K$ is called weakly ramified (tamely ramified, unramified), if $G_{s}=0$ for $s=2$ ( $s=1, s=0$, respectively).

The case $b=0$ of the following theorem is the well-known result of E. Noether that $L / K$ is tamely ramified if and only if $\mathcal{O}_{L}$ is free (of rank 1) over the group ring $\mathcal{O}_{K}[G]$ (see [No]). When $b=1$ the theorem implies that $L / K$ is weakly ramified if and only if $\mathfrak{m}_{L}$ is free over $\mathcal{O}_{K}[G]$. Furthermore it describes all fractional ideals in $L$ which are $\mathcal{O}_{K}[G]$-free. For example, if $L / K$ is not weakly ramified, then there does not exist any $\mathcal{O}_{K}[G]$-free fractional ideal in $L$ at all.

THEOREM 1.1. Let $b \in \mathbb{Z}$. Then the fractional ideal $\mathfrak{m}_{L}^{b}$ of $L$ is free over $\mathcal{O}_{K}[G]$ if and only if $L / K$ is weakly ramified and $b \equiv 1 \bmod \left|G_{1}\right|$.

Remark 1.2. (a) The only-if-part of Theorem 1.1 follows from Theorem 3 and the corollary of Proposition 2 in Ullom's paper [U13]. The tame case, i.e., $\left|G_{1}\right|=1$, and the case $b=1, G=G_{1}$ of the if-part of Theorem 1.1 are proved in Theorem 1 and Theorem 2 in his paper. Unfortunately, he does not state the general case of the if-part of Theorem 1.1 which is essential for this paper. Though it certainly can be proved with the methods he has developed in his papers [U11], [U12] and [U13], we here give a coherent and self-contained proof of Theorem 1.1 for the reader's convenience.

(b) In the geometric case, Pink has given a "global proof" for the fact that $L / K$ is weakly ramified if and only if $\mathfrak{m}_{L}$ is $\mathcal{O}_{K}[G]$-free (see Corollary 3.6 in [Pi]); to be precise, the if-direction is proved there only under the additional assumption $G=G_{1}$. Note that, in his terminology, weakly ramified means of type 2.

(c) Let $\operatorname{ord}(G)$ be odd. Then Theorem 1.1 also implies Erez' theorem that $L / K$ is weakly ramified if and only if the so-called square root of the inverse different is $\mathcal{O}_{K}[G]$-free (see Theorem 1 in [Er1]).

We will use the following proposition and lemma in the proof of Theorem 1.1. We recall that a $\mathbb{Z}[G]$-module $M$ is called cohomologically trivial, if the Tate cohomology groups $\hat{H}^{i}(U, M), i \in \mathbb{Z}$, vanish for all subgroups $U$ of $G$.

Proposition 1.3. Let $M$ be any $\mathcal{O}_{K}[G]$-module. Then $M$ is projective over $\mathcal{O}_{K}[G]$ if and only if $M$ is projective over $\mathcal{O}_{K}$ and cohomologically trivial.

Proof. If the ring of coefficients $\mathcal{O}_{K}$ is replaced by $\mathbb{Z}$, this is Théorème $7, \S 5$, Ch. IX on p. 151 in [Se1]. The same proof applies, if the ring of coefficients is any Dedekind domain. See also Proposition 4.1(a) on p. 457 in [Ch].

As usual, we denote the multiplication with the norm element $\sum_{\sigma \in G}[\sigma] \epsilon$ $\mathbb{Z}[G]$ by $\operatorname{Tr}_{L / K}$, the different of $L / K$ by $\mathcal{D}_{L / K}$, and the ramification index of $L / K$ by $e:=e_{L / K}$. Furthermore, for any $r \in \mathbb{R}$, the standard notation $\lfloor r\rfloor$ means the greatest integer less than or equal to $r$. 
LEMMA 1.4. Let $b \in \mathbb{Z}$. Then we have:

(a) $\left(\mathfrak{m}_{L}^{b}\right)^{G}=\mathfrak{m}_{K}^{a}$, where $a=1+\left\lfloor\frac{b-1}{e}\right\rfloor$.

(b) $\operatorname{Tr}_{L / K}\left(\mathfrak{m}_{L}^{b}\right)=\mathfrak{m}_{K}^{a^{\prime}}$, where $a^{\prime}=\left\lfloor\frac{\operatorname{ord}\left(\mathcal{D}_{L / K}\right)+b}{e}\right\rfloor$.

In particular, the Tate cohomology group $\hat{H}^{0}\left(G, \mathfrak{m}_{L}^{b}\right)$ vanishes if and only if $a=a^{\prime}$.

Proof. Let $a \in \mathbb{Z}$ and $a^{\prime} \in \mathbb{Z}$ be defined by $\left(\mathfrak{m}_{L}^{b}\right)^{G}=\mathfrak{m}_{K}^{a}$ and $\operatorname{Tr}_{L / K}\left(\mathfrak{m}_{L}^{b}\right)=\mathfrak{m}_{K}^{a^{\prime}}$, respectively. The obvious relation

$$
\mathfrak{m}_{L}^{a e}=\mathfrak{m}_{K}^{a} \mathcal{O}_{L} \subseteq \mathfrak{m}_{L}^{b} \subsetneq \mathfrak{m}_{K}^{a-1} \mathcal{O}_{L}=\mathfrak{m}_{L}^{(a-1) e}
$$

implies $a \geq \frac{b}{e}>a-1$, hence $a=1+\left\lfloor\frac{b-1}{e}\right\rfloor$. This proves assertion (a). Furthermore we have: $\mathcal{D}_{L / K}^{-1}=\left\{x \in L: \operatorname{Tr}_{L / K}\left(x \cdot \mathcal{O}_{L}\right) \subseteq \mathcal{O}_{K}\right\}$. Hence:

$$
\mathfrak{m}_{L}^{-e a^{\prime}+b}=\mathfrak{m}_{K}^{-a^{\prime}} \mathfrak{m}_{L}^{b} \subseteq \mathcal{D}_{L / K}^{-1} \subsetneq \mathfrak{m}_{K}^{-\left(a^{\prime}+1\right)} \mathfrak{m}_{L}^{b}=\mathfrak{m}_{L}^{-e\left(a^{\prime}+1\right)+b}
$$

Thus $-e a^{\prime}+b \geq-\operatorname{ord}\left(\mathcal{D}_{L / K}\right)>-e\left(a^{\prime}+1\right)+b$, i.e., $a^{\prime}=\left\lfloor\frac{\operatorname{ord}\left(\mathcal{D}_{L / K}\right)+b}{e}\right\rfloor$, as was to be shown.

Proof of Theorem 1.1. We first proof the if-part. We want to show that the fractional ideal $I:=\mathfrak{m}_{L}^{b}$ is free over $\mathcal{O}_{K}[G]$. Since $I \otimes L \cong L$ is free over $K[G]$ by the normal basis theorem, it suffices to show that $I$ is projective over $\mathcal{O}_{K}[G]$ by a theorem of Swan (see Corollary 6.4 on p. 567 in [Sw]). By Proposition 1.3, it then suffices to show that the Tate cohomology groups $\hat{H}^{i}\left(U, \mathfrak{m}_{L}^{b}\right), i \in \mathbb{Z}$, vanish for any subgroup $U$ of $G$. Since the extension $L / L^{U}$ is again weakly ramified and since the first ramification group $U_{1}$ of the extension $L / L^{U}$ is contained in $G_{1}$ (see Prop. $2, \S 1, \mathrm{Ch}$. IV on p. 70 in [Se1]), it suffices to consider the case $U=G$. The Hochschild-Serre spectral sequence for ordinary group (co)homology (supplemented by an obvious argument for Tate's 0th cohomology group $\hat{H}^{0}$ ) yields the following fact for any normal subgroup $N$ of $G$ : If $\hat{H}^{i}\left(N, \mathfrak{m}_{L}^{b}\right)=0$ and $\hat{H}^{i}\left(G / N,\left(\mathfrak{m}_{L}^{b}\right)^{N}\right)=0$ for all $i \in \mathbb{Z}$, then also $\hat{H}^{i}\left(G, \mathfrak{m}_{L}^{b}\right)=0$ for all $i \in \mathbb{Z}$. Applying this fact to the filtration $G \supseteq G_{0} \supseteq G_{1} \supseteq\{1\}$ of $G$, we are reduced to showing that $\hat{H}^{i}\left(G_{1}, \mathfrak{m}_{L}^{b}\right)=0$, that $\hat{H}^{i}\left(G_{0} / G_{1}, I\right)=0$ for any fractional ideal $I$ in $L^{G_{1}}$ and that $\hat{H}^{i}\left(G / G_{0}, J\right)=0$ for any fractional ideal $J$ in $L^{G_{0}}$ (for all $i \in \mathbb{Z}$ ). Applying this fact again to a filtration of the abelian group $G_{1}$ (of exponent $p$ ) whose successive quotients are cyclic groups of order $p$, we see that it finally suffices to show that $\hat{H}^{i}\left(G, \mathfrak{m}_{L}^{b}\right)=0$ for all $i \in \mathbb{Z}$ and $b \in \mathbb{Z}$ with $b \equiv 1 \bmod \left|G_{1}\right|$ in the following three cases (note that, for any subgroup $U$ of $G_{1}$ of order $p$, we have $\left(\mathfrak{m}_{L}^{b}\right)^{U}=\mathfrak{m}_{L^{U}}^{a}$ with $a \equiv 1 \bmod \left|G_{1} / U\right|$ by Lemma $1.4(\mathrm{a})$ ):

(i) $G_{1}$ is cyclic of order $p$ and $G=G_{1}$, i.e., $L / K$ is totally wildly ramified of order $p$.

(ii) $G_{1}=\{1\}$ and $G=G_{0}$, i.e., $L / K$ is totally tamely ramified.

(iii) $G_{0}=1$, i.e., $L / K$ is unramified. 
We first consider the case (i). Since $G$ is cyclic, it suffices to show $\hat{H}^{i}\left(G, \mathfrak{m}_{L}^{b}\right)=$ 0 for $i=0$ and $i=1$. Using Hilbert's formula for the order of the different (see Prop. $4, \S 1$, Ch. IV on p. 72 in [Se1]) and the congruence $b \equiv 1 \bmod p$, we obtain:

$$
\left\lfloor\frac{\operatorname{ord}\left(\mathcal{D}_{L / K}\right)+b}{p}\right\rfloor=\left\lfloor\frac{2(p-1)+b}{p}\right\rfloor=1+\left\lfloor\frac{b-1}{p}\right\rfloor .
$$

Hence, by Lemma (1.4), we have $\hat{H}^{0}\left(G, \mathfrak{m}_{L}^{b}\right)=0$. To show $\hat{H}^{1}\left(G, \mathfrak{m}_{L}^{b}\right)=0$, it suffices to show that the Herbrand difference

$$
h\left(\mathfrak{m}_{L}^{b}\right):=\operatorname{length}_{\mathcal{O}_{K}}\left(\hat{H}^{0}\left(G, \mathfrak{m}_{L}^{b}\right)\right)-\operatorname{length}_{\mathcal{O}_{K}}\left(\hat{H}^{1}\left(G, \mathfrak{m}_{L}^{b}\right)\right)
$$

vanishes. Since $\mathfrak{m}_{L}^{b} \otimes \mathcal{O}_{K} K \cong L$ is $K[G]$-free of rank 1 , we can find a free $\mathcal{O}_{K}[G]$ submodule $M$ of $\mathfrak{m}_{L}^{b}$ such that $\mathfrak{m}_{L}^{b} / M$ is of finite length. Now $h\left(\mathfrak{m}_{L}^{b}\right)=0$ follows from the well-known (and easy) facts that the Herbrand difference vanishes for any free $\mathcal{O}_{K}[G]$-module and for any $\mathcal{O}_{K}[G]$-module of finite length, and that the Herbrand difference is additive on short exact sequences (see $\S 7$, Chapter IV of Neukirch's book [Ne] where the multiplicative version of the Herbrand difference, the Herbrand quotient, is treated in detail).

In the cases (ii) and (iii), we have to show that $\hat{H}^{i}(G, I)=0$ (for all $i \in \mathbb{Z}$ ) for any arbitrary fractional ideal $I$ of $L$. Let $\pi_{K}$ be a prime element in $\mathcal{O}_{K}$. Then we have $\hat{H}^{i}\left(G, I / \pi_{K} I\right)=0$ for all $i$. Indeed, in the case (ii) this is a consequence of Corollaire $1, \S 2$, Ch. VIII on p. 138 in [Se1], since $I / \pi_{K} I$ is annihilated by a power of $p$ and the order of $G$ is prime to $p$; in the case (iii) it is a consequence of the classical normal basis theorem and of Proposition (1.3), since $I / \pi_{K} I$ is isomorphic to $\lambda$ as a $\kappa[G]$-module. The long exact sequence associated with the short exact sequence of $G$-modules

$$
0 \rightarrow I \stackrel{\pi_{K}}{\rightarrow} I \rightarrow I / \pi_{K} I \rightarrow 0
$$

now shows that $\pi_{K} \hat{H}^{i}(G, I)=\hat{H}^{i}(G, I)$ for all $i \in \mathbb{Z}$. Finally, Nakayama's Lemma implies that $\hat{H}^{i}(G, I)=0$ for all $i \in \mathbb{Z}$. This completes the proof of the if-direction in Theorem 1.1.

We now prove the only-if-direction. So, let $b \in \mathbb{Z}$ such that $\mathfrak{m}_{L}^{b}$ is $\mathcal{O}_{K}[G]$-free. We first show that $L / K$ is weakly ramified. Let $s$ be the greatest integer such that $G_{s} \neq\{1\}$. We may assume that $s \geq 1$. Then there is a cyclic subgroup $U$ of $G_{s}$ of order $p$. By Proposition (1.3) we have $\hat{H}^{0}\left(U, \mathfrak{m}_{L}^{b}\right)=0$, i.e., $\operatorname{Tr}_{L / L^{U}}\left(\mathfrak{m}_{L}^{b}\right)=\left(\mathfrak{m}_{L}^{b}\right)^{U}$. Furthermore, we have ord $\left(\mathcal{D}_{L / L^{U}}\right)=(s+1)(p-1)$ by Prop. 2 and Prop. 4 of $\S 1$, Ch. IV in [Se1]. Now Lemma 1.4 applied to the extension $L / L^{U}$ implies that

$$
s+1+\left\lfloor\frac{b-1-s}{p}\right\rfloor=\left\lfloor\frac{(s+1)(p-1)+b}{p}\right\rfloor=1+\left\lfloor\frac{b-1}{p}\right\rfloor .
$$


Hence $s$ must be equal to 1 , i.e., $L / K$ is weakly ramified. It remains to show that $b \equiv 1 \bmod \left|G_{1}\right|$. By Proposition 1.3 we have $\hat{H}^{0}\left(G_{1}, \mathfrak{m}_{L}^{b}\right)=0$, i.e., $\operatorname{Tr}_{L / L^{G_{1}}}\left(\mathfrak{m}_{L}^{b}\right)=\left(\mathfrak{m}_{L}^{b}\right)^{G_{1}}$. Furthermore, we have $\operatorname{ord}\left(\mathcal{D}_{L / L^{G_{1}}}\right)=2 \cdot\left(\left|G_{1}\right|-1\right)$ by Prop $4, \S 1$, Ch. IV on p. 72 in [Se1]. Now, Lemma 1.4 applied to the extension $L / L^{G_{1}}$ implies:

$$
2+\left\lfloor\frac{b-2}{\left|G_{1}\right|}\right\rfloor=1+\left\lfloor\frac{b-1}{\left|G_{1}\right|}\right\rfloor .
$$

Hence $b \equiv 1 \bmod \left|G_{1}\right|$, as desired. Thus, the proof of Theorem 1.1 is complete.

2. Projectivity of Zariski cohomology. Let $k$ be an algebraically closed field of characteristic $p>0, X$ a connected smooth projective curve over $k$ and $G$ a finite subgroup of the automorphism group $\operatorname{Aut}(X / k)$ of order $n$.

In this section we give both sufficient and necessary conditions under which the Zariski cohomology groups of $X$ with values in a $G$-equivariant invertible $\mathcal{O}_{X}$-module are projective over the group ring $k[G]$.

Let $\pi: X \rightarrow Y:=X / G$ denote the canonical projection, and let $g_{X}$ resp. $g_{Y}$ denote the genus of $X$ resp. $Y$. Furthermore, for any $P \in X$, we denote the decomposition group $\{\sigma \in G: \sigma(P)=P\}$ by $G_{P}$, the ramification index of $\pi$ at the place $P$ by $e_{P}$, the higher ramification groups (see Chapitre IV in [Se1]) by $G_{P, s}, s \geq 0$, the wild part of the ramification index, i.e. $\left|G_{P, 1}\right|$, by $e_{P}^{\mathrm{W}}$ and the tame part of the ramification index, i.e. $\left|G_{P} / G_{P, 1}\right|$, by $e_{P}^{\mathrm{t}}$. We say that $\pi$ is weakly ramified, if $G_{P, s}$ is trivial for $s \geq 2$ and all $P \in X$.

We denote the Grothendieck group of all finitely generated $k[G]$-modules (resp., of all finitely generated projective $k[G]$-modules) by $K_{0}(G, k)$ (resp., by $\left.K_{0}(k[G])\right)$. We recall from classical representation theory that the set of isomorphism classes of irreducible $k[G]$-modules (resp., of indecomposable projective $k[G]$-modules) forms a basis of $K_{0}(G, k)$ (resp., of $\left.K_{0}(k[G])\right)$ and that the Cartan homomorphism $K_{0}(k[G]) \rightarrow K_{0}(G, k)$ is injective (see Chapters 14 and 16 in Serre's book $[\mathrm{Se} 2])$.

We recall that a locally free $G$-module on $X$ is a locally free $\mathcal{O}_{X}$-module $\mathcal{E}$ together with $\mathcal{O}_{X}$-isomorphisms $g^{*}(\mathcal{E}) \rightarrow \mathcal{E}, g \in G$, which satisfy the usual composition rules. For instance, if $D=\sum_{P \in X} n_{P}[P]$ is a $G$-equivariant divisor on $X$ (i.e., $n_{\sigma(P)}=n_{P}$ for all $\sigma \in G$ and $\left.P \in X\right)$, then the $\mathcal{O}_{X}$-module $\mathcal{O}_{X}(D)$ is a locally free $G$-module on $X$ of rank 1 . The Zariski cohomology groups $H^{i}(X, \mathcal{E})$, $i \geq 0$, are then $k$-representations of $G$ in the obvious way. Let

$$
\chi(G, X, \mathcal{E}):=\left[H^{0}(X, \mathcal{E})\right]-\left[H^{1}(X, \mathcal{E})\right] \in K_{0}(G, k)
$$

denote the equivariant Euler characteristic of $X$ with values in $\mathcal{E}$.

In Theorem 2 of [Na3], Nakajima proved that the cover $\pi$ is tamely ramified if and only if there exists a divisor $D=\sum_{P \in X} n_{P}[P]$ on $X$ of the form $D=\pi^{*}(E), E$ 
any divisor on $Y$, with $\operatorname{deg}(D)>2 g_{X}-2$ such that the $k[G]$-module $H^{0}\left(X, \mathcal{O}_{X}(D)\right)$ is projective. The following theorem generalizes this statement. (Note that for any divisor $D=\sum_{P \in X} n_{P}[P]$ on $X$ of the form $D=\pi^{*}(E)$, we have $n_{P} \equiv 0 \bmod e_{P}$ for all $P \in X$.)

THEOREM 2.1. Let $D=\sum_{P \in X} n_{P}[P]$ be a G-equivariant divisor on $X$.

(a) If $\pi$ is weakly ramified and $n_{P} \equiv-1 \bmod e_{P}^{\mathrm{W}}$ for all $P \in X$, then the equivariant Euler characteristic $\chi\left(G, X, \mathcal{O}_{X}(D)\right) \in K_{0}(G, k)$ lies in the image of the Cartan homomorphism $K_{0}(k[G]) \rightarrow K_{0}(G, k)$. If moreover one of the two cohomology groups $H^{i}\left(X, \mathcal{O}_{X}(D)\right), i=0,1$, vanishes, then the other one is a projective $k[G]$-module.

(b) Let $\operatorname{deg}(D)>2 g_{X}-2$. If the $k[G]$-module $H^{0}\left(X, \mathcal{O}_{X}(D)\right)$ is projective, then $\pi$ is weakly ramified and $n_{P} \equiv-1 \bmod e_{P}^{\mathrm{W}}$ for all $P \in X$.

Proof. (a) Theorem 1.1 implies that, for any $P \in X$, the $\mathcal{O}_{Y, \pi(P)}\left[G_{P}\right]$-module $\mathcal{O}_{X}(D)_{P}=\mathfrak{m}_{P}^{-n_{P}}$ is free after completion. From Corollary (76.9) on p. 533 in [CR1] we obtain that this is even true without completion. Hence the direct image $\pi_{*}\left(\mathcal{O}_{X}(D)\right)$ is a locally free $\mathcal{O}_{Y}[G]$-module. Furthermore we have $H^{i}\left(X, \mathcal{O}_{X}(D)\right)=H^{i}\left(Y, \pi_{*}\left(\mathcal{O}_{X}(D)\right)\right)$ for all $i \in \mathbb{Z}$. Now Theorem 1.1 on p. 447 and Proposition 4.1(a) on p. 457 in Chinburg's paper [Ch] imply that there exists a bounded complex $L^{*}$ of finitely generated projective $k[G]$-modules such that the $k[G]$-module $H^{i}\left(X, \mathcal{O}_{X}(D)\right)$ is isomorphic to the $i$ th cohomology module $H^{i}\left(L^{*}\right)$ for all $i \in \mathbb{Z}$. This fact immediately implies the first assertion of Theorem 2.1(a). The second assertion can be derived from this fact as in the proof of Theorem 2 in Nakajima's paper [Na1].

(b) We first prove this in the case that $G$ is cyclic of order $p$. We fix a point $P \in X$. Let $M$ denote the greatest integer such that $G_{P, M}$ is not trivial. We may assume that $M \geq 1$. By definition of the higher ramification groups we have $M+1=\operatorname{ord}_{P}(\sigma(x)-x)$ where $\sigma \in G \backslash\{1\}$ and $x$ is any prime element of the local ring $\mathcal{O}_{X, P}$. Since the $k[G]$-module $H^{0}\left(X, \mathcal{O}_{X}(D)\right)$ is projective, the multiplicities of the non-projective indecomposable $k[G]$-modules in $H^{0}\left(X, \mathcal{O}_{X}(D)\right)$ are zero. By Theorem 1 on p. 86 in [Na3], these multiplicities are given by

$$
m_{j}:=\frac{M}{p}+\left\langle\frac{n_{P}-j M}{p}\right\rangle-\left\langle\frac{n_{P}-(j-1) M}{p}\right\rangle, \quad j=1, \ldots, p-1 ;
$$

here, for a rational number $a$, $\langle a\rangle$ denotes the fractional part of $a$, i.e., $0 \leq\langle a\rangle<1$ and $a-\langle a\rangle$ is an integer. Since $M$ is not divisible by $p$ (see Lemma 1 on p. 87 in [Na3]), there is a solution $j_{0} \in\{0, \ldots, p-1\}$ of the congruence $n_{P} \equiv\left(j_{0}-1\right) M$ $\bmod p$. If $j_{0} \neq 0$, then one of the integers $m_{j}, j=1, \ldots, p-1$, would be positive, namely $m_{j_{0}}=\frac{M}{p}+\left\langle\frac{n_{P}-j_{0} M}{p}\right\rangle$. Hence $j_{0}=0$, i.e., $M \equiv-n_{P} \bmod p$; therefore

$$
m_{j}=\frac{M}{p}+\left\langle\frac{-j M}{p}-\frac{M}{p}\right\rangle-\left\langle\frac{-j M}{p}\right\rangle \quad \text { for all } j=1, \ldots, p-1 .
$$


Let now $j_{1} \in\{1, \ldots, p-1\}$ be a solution of the congruence $-j_{1} M \equiv 1 \bmod p$. If $M \not \equiv 1 \bmod p$, then

$$
m_{j_{1}}=\frac{M}{p}+\left\langle\frac{1}{p}-\frac{M}{p}\right\rangle-\frac{1}{p}=\frac{M}{p}+1-\left\langle\frac{M}{p}\right\rangle
$$

would be positive. Hence $M \equiv 1 \bmod p$; therefore

$$
m_{j}=\frac{M}{p}+\left\langle\frac{-j-1}{p}\right\rangle-\left\langle\frac{-j}{p}\right\rangle=\frac{M}{p}+\frac{p-j-1}{p}-\frac{p-j}{p}=\frac{M-1}{p}
$$

for all $j=1, \ldots, p-1$. Hence we have $M=1$ and $n_{P} \equiv-1 \bmod p$, as was to be shown.

We now consider the general case. We fix a point $P \in X$ with $e_{P}^{\mathrm{W}} \neq 1$. (If such a point does not exist, we are done.) Let $M$ denote the greatest integer such that $G_{P, M}$ is not trivial. We choose a cyclic subgroup $H$ of $G_{P, M}$ of order $p$. Let $\eta: X \rightarrow Z:=X / H$ denote the corresponding cover. Since $H^{0}\left(X, \mathcal{O}_{X}(D)\right)$ is also projective as a $k[H]$-module and since, for any $s \geq 0$, the intersection $G_{P, s} \cap H$ is the sth ramification group of the cover $\eta$ at $P$ (see Prop. 2, $\S 1$, Ch. IV on p. 70 in [Se1]), we obtain from the case considered above that $M=1$ and that $n_{P^{\prime}} \equiv-1 \bmod f_{P^{\prime}}$ for all $P^{\prime} \in X$ (where $f_{P^{\prime}}$ denotes the ramification index of $\eta$ at $\left.P^{\prime}\right)$. It remains to show that $n_{P} \equiv-1 \bmod e_{P}^{\mathrm{w}}$. We prove this by induction on $n:=\operatorname{ord}(G)$. The case $n=1$ is trivial. Let $g_{Z}$ denote the genus of $Z$, and let the divisor $E$ on $Z$ be defined by the equality $\mathcal{O}_{Z}(E)=\eta_{*}^{H}\left(\mathcal{O}_{X}(D)\right)$ of subsheaves of the constant sheaf $K(Z)$, the function field of $Z$; here, $\eta_{*}^{H}\left(\mathcal{O}_{X}(D)\right)$ denotes the subsheaf of the direct image $\eta_{*}\left(\mathcal{O}_{X}(D)\right)$ fixed by the obvious action of $H$. Then by Lemma 1.4(a), the multiplicity of $E$ at any point $Q \in Z$ is $-\left(1+\left\lfloor\frac{-n_{Q}-1}{f_{Q}}\right\rfloor\right)=-1+\frac{n_{Q^{+1}}}{f_{Q}}$ where $n_{Q}:=n_{\tilde{Q}}$ and $f_{Q}:=f_{\tilde{Q}}$ for any point $\tilde{Q} \in X$ in the fibre $\eta^{-1}(Q)$. Thus we have:

$$
\begin{aligned}
p \cdot \operatorname{deg}(E) & =p \cdot \sum_{Q \in Z}\left(-1+\frac{n_{Q}+1}{f_{Q}}\right) \\
& =\sum_{P^{\prime} \in X}\left(-f_{P^{\prime}}+n_{P^{\prime}}+1\right) \\
& =\operatorname{deg}(D)-\sum_{P^{\prime} \in X}\left(f_{P^{\prime}}-1\right) \\
& \geq 2\left(g_{X}-1\right)-\sum_{P^{\prime} \in X} 2\left(f_{P^{\prime}}-1\right) \\
& \geq p \cdot\left(2 g_{Z}-2\right)
\end{aligned}
$$

by Hurwitz' formula (see Corollary 2 on p. 301 in [Ha]) and Hilbert's formula (see Prop. $4, \S 1$, Ch. IV on p. 72 in [Se1]). Furthermore $H^{0}\left(Z, \mathcal{O}_{Z}(E)\right)=$ $H^{0}\left(X, \mathcal{O}_{X}(D)\right)^{H}$ is projective as a $k\left[G_{P, 1} / H\right]$-module. Now the induction hypoth- 
esis applied to the Galois cover $Z \rightarrow X / G_{P, 1}$ (with Galois group $G_{P, 1} / H$ ) and to the divisor $E$ on $Z$ implies that the multiplicity $-1+\frac{n_{P}+1}{p}$ of $E$ at $\eta(P)$ is congruent to -1 modulo $\frac{e_{P}^{\mathrm{W}}}{p}$, hence $n_{P} \equiv-1 \bmod e_{P}^{\mathrm{W}}$, as was to be shown. This completes the proof of Theorem 2.1.

Question 2.2. Recall that a bounded complex of finitely generated projective $k[G]$-modules is called a perfect complex. The proof of Theorem 2.1(a) in fact yields the following stronger result: If $\pi$ is weakly ramified and $n_{P} \equiv-1 \bmod e_{P}^{\mathrm{W}}$ for all $P \in X$, then $R \Gamma\left(X, \mathcal{O}_{X}(D)\right)$ is quasi-isomorphic to a perfect complex. If $\operatorname{deg}(D)>2 g_{X}-2$, then Theorem 2.1(b) shows that also the converse statement is true. Is the converse statement true in general?

COROLlary 2.3. Let $S$ be any non-empty G-stable set of closed points on $X$ containing all ramified points, and let $\Omega_{X}(S):=\Omega_{X} \otimes \mathcal{O}_{X}\left(\sum_{P \in S}[P]\right)$ denote the sheaf of meromorphic differentials on $X$ which are logarithmic along $S$. Then, $\pi$ is weakly ramified if and only if the $k[G]$-module $H^{0}\left(X, \Omega_{X}(S)\right)$ is projective.

Proof. By Théorème 2.34 on p. 44 in [Bo1], there is a $G$-equivariant divisor $K_{X}=\sum_{P \in X} n_{P}^{\prime}[P]$ on $X$ such that $\mathcal{O}_{X}\left(K_{X}\right) \cong \Omega_{X}$. Then the divisor $D=K_{X}+$ $\sum_{P \in S}[P]$ satisfies $\operatorname{deg}(D)>2 g_{X}-2$ (by Example 1.3.3 on p. 296 in [Ha]). Hence, Theorem 2.1(b) implies the if-part of Corollary 2.3. We now prove the only-if-part. We have a natural short exact sequence

$$
0 \rightarrow \pi^{*}\left(\Omega_{Y}\right) \rightarrow \Omega_{X} \rightarrow \Omega_{X / Y} \rightarrow 0
$$

of coherent $G$-modules on $X$. Let the divisor $K_{Y}=\sum_{Q \in Y} r_{Q}[Q]$ on $Y$ be defined by the equality $\pi^{*}\left(\Omega_{Y}\right)=\mathcal{O}_{X}\left(\pi^{*}\left(K_{Y}\right)\right)$ of subsheaves of $\mathcal{O}_{X}\left(K_{X}\right)$. If $\pi$ is weakly ramified, Hilbert's formula (see Prop. 4, $\S 1$, Ch. IV on p. 72 in [Se1]) then implies that $n_{P}^{\prime}-e_{P} r_{\pi(P)}=\left(e_{P}-1\right)+\left(e_{P}^{\mathrm{W}}-1\right)$ for all $P \in X$. In particular, the divisor $D$ satisfies the condition of Theorem 2.1(a), and Theorem 2.1(a) finally implies the only-if-part of Corollary 2.3.

Remark 2.4. Let $S$ and $\Omega_{X}(S)$ be as in Corollary 2.3. Kani states in Theorem 1 of [Ka] that $H^{0}\left(X, \Omega_{X}(S)\right)$ is a projective $k[G]$-module if and only if $\pi$ is tamely ramified. Since there exist weakly ramified covers which are not tamely ramified (see the case $r=1$ in Example 2.5 below), the only-if-direction of Corollary 2.3 contradicts the only-if-direction of Kani's result which seems to be wrong. (More precisely, the final displayed equation and the computation of $m_{y}$ for $y \in Y_{\text {ram }}$ in the proof of the only-if-part of that theorem seem to be wrong.)

The following example (taken from Hasse's paper [Has]) shows that every theoretically possible "type" of ramification for Galois covers of smooth projec- 
tive curves can occur. In this example, the base curve is the projective line, the Galois group is cyclic of order $p$, and only one point is ramified.

Example 2.5. Let $r \in \mathbb{N}$ such that $p$ does not divide $r$. Let $k(x, y)$ be the cyclic field extension of the rational function field $k(x)$ of degree $p$ given by the Artin-Schreier equation $y^{p}-y=x^{r}$. Let $\pi: C \rightarrow \mathbb{P}_{k}^{1}$ denote the corresponding cover of nonsingular curves. Then $\pi$ is unramified precisely over $\mathbb{A}_{k}^{1} \subset \mathbb{P}_{k}^{1}$, and, at the unique point $P \in C$ lying over $\infty \in \mathbb{P}_{k}^{1}$, the greatest integer $M$ such that $G_{P, M}$ is not trivial is equal to $r$. Furthermore, the genus of $C$ is equal to $\frac{(r-1)(p-1)}{2}$.

Proof. The first assertion is proved in [Has]. The genus $g_{C}$ of $C$ is determined by the formula $2\left(g_{C}-1\right)=-2 p+(M+1)(p-1)$ which is a consequence of Hurwitz' formula (see Corollary 2.4 on p. 301 in [Ha]) and Hilbert's formula (see Prop. $4, \S 1$, Ch. IV on p. 72 in [Se1]). Hence $g_{C}=\frac{(r-1)(p-1)}{2}$, as was to be shown.

3. Computing equivariant Euler characteristics. We keep the assumptions and notations introduced in Section 2.

In [EL], Ellingsrud and Lønsted have proved a formula for the equivariant Euler characteristic $\chi(G, X, \mathcal{E}) \in K_{0}(G, k)$ of $X$ with values in any locally free $G$-module $\mathcal{E}$ on $X$, if $(\operatorname{char}(k), n)=1$. In [Kö2], using a different approach, we have given a simplified version of their formula which holds also in the tamely ramified case. In this section we generalize that formula to the case of an arbitrary cover $\pi$.

In particular, we do not assume any condition on the ramification of the cover $\pi$ in this section. As just explained, the main focus in this paper is on the case $p=\operatorname{char}(k)>0$, but everything in this and the following section is true and interesting (though classical) also in the case $p=0$ (if the condition prime to $p$ is regarded as the empty condition and the term $p$-group means trivial group).

Let $\mathcal{E}$ be a locally free $G$-module on $X$ of rank $r$. For any $P \in X$, we view the fibre $\mathcal{E}(P):=\mathcal{E}_{P} / \mathfrak{m}_{P} \mathcal{E}_{P}$ as a $k$-representation of the decomposition group $G_{P}$. Furthermore, the obvious representation of $G_{P}$ on the cotangent space $\mathfrak{m}_{P} / \mathfrak{m}_{P}^{2}$ (or the corresponding character $G_{P} \rightarrow k^{\times}$) is denoted by $\chi_{P}$.

The following theorem computes the equivariant Euler characteristic $\chi(G, X$, $\mathcal{E}) \in K_{0}(G, k)$ in terms of the rank and degree of $\mathcal{E}$, the genus $g_{Y}$ of $Y:=X / G$, the order $n$ of $G$, the orders of the higher ramification groups of $\pi$, and the representations of decomposition groups on the fibres of $\mathcal{E}$ and on the cotangent spaces.

THEOREM 3.1. We have

(1) $\chi(G, X, \mathcal{E})=c \cdot[k[G]]-\frac{1}{n} \sum_{P \in X} e_{P}^{\mathrm{w}} \sum_{d=1}^{e_{P}^{\mathrm{t}}-1} d \cdot\left[\operatorname{Ind}_{G_{P}}^{G}\left(\mathcal{E}(P) \otimes \chi_{P}^{d}\right)\right]$ in $K_{0}(G, k)_{\mathbb{Q}}$ 
where

$$
c=\left(1-g_{Y}\right) r+\frac{1}{n} \operatorname{deg}(\mathcal{E})-\frac{r}{2 n} \sum_{P \in X}\left(\left(e_{P}^{\mathrm{w}}-1\right)\left(e_{P}^{\mathrm{t}}+1\right)+\sum_{s \geq 2}\left(\left|G_{P, s}\right|-1\right)\right) .
$$

Remark 3.2. (a) If $\pi$ is tamely ramified, then the sum over $P \in X$ in the definition of the multiplicity $c$ obviously vanishes. In particular, Theorem 3.1 generalizes Theorem 1.1 in [Kö2]. In the next section, we will apply Theorem 3.1 in the weakly ramified case, i.e., when the sum $\sum_{s \geq 2}\left(\left|G_{P, s}\right|-1\right)$ vanishes for all $P \in X$

(b) Using the Hurwitz formula, see Corollary 2.4 on p. 301 in [Ha], one can easily simplify the expression for the multiplicity $c$ as follows:

$$
c=\frac{1}{n}\left(\left(1-g_{X}\right) r+\operatorname{deg}(\mathcal{E})-\frac{r}{2} \sum_{P \in X}\left(e_{P}^{\mathrm{t}}-1\right)\right)
$$

(see also the proof of Theorem 3.1 below). However the applications of the formula (1) rely on the more complicated form as stated in Theorem 3.1.

(c) The reader may wish to compare Theorem 3.1 with Théorème 3.18 in Borne's paper [Bo2] which gives an alternative expression for $\chi(G, X, \mathcal{E})$.

Proof of Theorem 3.1. By classical representation theory (see Corollary 17.10 on p. 424 in [CR2]) it suffices to show that the Brauer characters of both sides of formula (1) coincide. For any $k$-representation $V$ of $G$ and for any $\sigma \in G$ of order prime to $p$ we write $\operatorname{Trace}(\sigma \mid V)$ for the value of the Brauer character of $V$ at $\sigma$. Recall that

$$
\operatorname{Trace}(\sigma \mid V)=\sum_{i=1}^{\operatorname{dim}(V)} \varphi\left(\alpha_{i}\right)
$$

where $\alpha_{i}, i=1, \ldots, \operatorname{dim}(V)$, are the eigenvalues of the $k$-linear map $\sigma$ on $V$ and $\varphi: k^{\times} \rightarrow K^{\times}$is the Teichmüller character from the group of invertible elements in $k$ to the group of invertible elements in the quotient field $K:=Q(W(k))$ of the Witt ring $W(k)$ of $k$. (We set $K:=k$ and $\varphi:=$ id, if $p=0$.)

We first show that the character values at $\sigma=1$, i.e., the $k$-dimensions, coincide. The $k$-dimension of the right-hand of (1) side is

$$
\begin{aligned}
n\left(1-g_{Y}\right) r+\operatorname{deg}(\mathcal{E})-\frac{r}{2} \sum_{P \in X}\left(\left(e_{P}^{\mathrm{w}}-1\right)\left(e_{P}^{\mathrm{t}}+1\right)+\right. & \left.\sum_{s \geq 2}\left(\left|G_{P, S}\right|-1\right)\right) \\
& -\frac{1}{n} \sum_{P \in X} e_{P}^{\mathrm{w}} \sum_{d=1}^{e_{P}^{\mathrm{t}}-1} d \cdot r \cdot \frac{n}{e_{P}} .
\end{aligned}
$$


By Hurwitz' formula (see Corollary 2.4 on p. 301 in [Ha]) and Hilbert's formula for the order of the different (see Prop. $4, \S 1$, Ch. IV on p. 72 in [Se1]), this is equal to

$$
\begin{aligned}
(1- & \left.g_{X}\right) r+\frac{r}{2} \sum_{P \in X} \sum_{s \geq 0}\left(\left|G_{P, s}\right|-1\right)+\operatorname{deg}(\mathcal{E}) \\
& -\frac{r}{2} \sum_{P \in X}\left(\left(e_{P}^{\mathrm{w}} e_{P}^{\mathrm{t}}-e_{P}^{\mathrm{t}}+e_{P}^{\mathrm{w}}-1\right)+\sum_{s \geq 2}\left(\left|G_{P, S}\right|-1\right)\right) \\
& -\sum_{P \in X} \frac{r}{e_{P}^{\mathrm{t}}} \cdot \frac{e_{P}^{\mathrm{t}}\left(e_{P}^{\mathrm{t}}-1\right)}{2} \\
= & \left(1-g_{X}\right) r+\operatorname{deg}(\mathcal{E})+\frac{r}{2} \sum_{P \in X}\left(e_{P}^{\mathrm{t}}-1\right) \\
& -\frac{r}{2} \sum_{P \in X}\left(e_{P}^{\mathrm{t}}-1\right) \\
= & \left(1-g_{X}\right) r+\operatorname{deg}(\mathcal{E}) .
\end{aligned}
$$

This is equal to the $k$-dimension of the left-hand side of (1) by the theorem of Riemann-Roch (see $\S 1$ in Chapter IV of [Ha] and Exercise 6.11 on p. 149 in $[\mathrm{Ha}])$.

We now fix an element $\sigma \in G \backslash\{1\}$ whose order is prime to $p$ and show that the character values of both sides of (1) coincide at $\sigma$. Using $\operatorname{Trace}(\sigma \mid k[G])=$ 0 and, more generally, the well-known formula for an induced character (see Lemma 21.28 on p. 509 in [CR2]), we obtain for the character value of the right hand side of (1) at $\sigma$ :

$$
\begin{aligned}
& -\frac{1}{n} \sum_{P \in X} e_{P}^{\mathrm{w}} \sum_{d=1}^{e_{P}^{\mathrm{t}}-1} d \cdot \operatorname{Trace}\left(\sigma \mid \operatorname{Ind}_{G_{P}}^{G}\left(\mathcal{E}(P) \otimes \chi_{P}^{d}\right)\right) \\
& =-\frac{1}{n} \sum_{P \in X} \sum_{d=1}^{e_{P}^{\mathrm{t}}-1} \frac{e_{P}^{\mathrm{W}} \cdot d}{e_{P}} \sum_{\tau \in G, \tau^{-1}}{ }_{\sigma \tau \in G_{P}} \operatorname{Trace}\left(\tau^{-1} \sigma \tau \mid \mathcal{E}(P)\right) \cdot \operatorname{Trace}\left(\tau^{-1} \sigma \tau \mid \chi_{P}^{d}\right) \\
& =-\frac{1}{n} \sum_{P \in X} \sum_{d=1}^{e_{P}^{\mathrm{t}}-1} \frac{d}{e_{P}^{\mathrm{t}}} \sum_{\tau \in G, \sigma \in G_{\tau(P)}} \operatorname{Trace}(\sigma \mid \mathcal{E}(\tau(P))) \cdot \operatorname{Trace}\left(\sigma \mid \chi_{\tau(P)}^{d}\right) \\
& =-\sum_{P \in X^{\sigma}} \operatorname{Trace}(\sigma \mid \mathcal{E}(P)) \cdot \frac{1}{e_{P}^{\mathrm{t}}} \sum_{d=1}^{e_{P}^{\mathrm{t}}-1} d \cdot \operatorname{Trace}\left(\sigma \mid \chi_{P}^{d}\right),
\end{aligned}
$$

where $X^{\sigma}:=\{P \in X: \sigma(P)=P\}$. Since $G_{P, 1}$ is a $p$-group (see Corollaire 3, $\S 2$, Ch. IV on p. 75 in [Se1]), the character $\chi_{P}: G_{P} \rightarrow k^{\times}$factors modulo $G_{P, 1}$, and the induced character $\bar{\chi}_{P}: G_{P} / G_{P, 1} \rightarrow k^{\times}$is injective by Corollaire $1, \S 2$, Ch. IV on p. 75 in [Se1] (for all $P \in X$ ). Hence $\chi_{P}(\sigma) \in k$ is a nontrivial $e_{P}^{\mathrm{t}}$ th root of 
unity and we obtain

$$
-\frac{1}{e_{P}^{\mathrm{t}}} \sum_{d=1}^{e_{P}^{\mathrm{t}}-1} d \cdot \operatorname{Trace}\left(\sigma \mid \chi_{P}^{d}\right)=\left(1-\operatorname{Trace}\left(\sigma \mid \chi_{P}\right)\right)^{-1}
$$

by Lemma 3.3 below. Thus the character value of the right-hand side of (1) at $\sigma$ is equal to

$$
\sum_{P \in X^{\sigma}} \frac{\operatorname{Trace}(\sigma \mid \mathcal{E}(P))}{1-\operatorname{Trace}\left(\sigma \mid \chi_{P}\right)}
$$

which in turn is equal to the character value of the left-hand side of (1) at $\sigma$ by the Lefschetz fixed point formula (see Chapter VI, $\S 9$ in [FL] or Example 3 in [Kö1]). This completes the proof of Theorem 3.1.

The following easy lemma also is a crucial step in an alternative approach to Theorem 5.2 of [Ch], see section $2 \mathrm{a}$ of [Er2].

Lemma 3.3. Let $m \in \mathbb{N}$ and $\zeta \neq 1$ an mth root of unity in any field. Then we have:

$$
m(\zeta-1)^{-1}=\sum_{d=1}^{m-1} d \zeta^{d}
$$

Proof. $\left(\sum_{d=1}^{m-1} d \zeta^{d}\right)(\zeta-1)=\sum_{d=1}^{m-1} d \zeta^{d+1}-\sum_{d=1}^{m-1} d \zeta^{d}=(m-1) \zeta^{m}-\sum_{d=1}^{m-1} \zeta^{d}=$ $m$.

The following lemma will be used in the proof of Lemma 3.5, a fact we will frequently use.

Lemma 3.4. Let $H$ be the semi-direct product of a finite p-group $P$ and an (arbitrary) group $C$ which acts on $P$. Then we have in $K_{0}(H, k)$ :

$$
[k[H]]=|P| \cdot[k[C]] .
$$

Proof. Let $I$ denote the augmentation ideal of the group ring $k[P]$. The group $C$ acts on $k[P]$ in the obvious way, and the ideals $I^{r}, r \geq 0$, of $k[P]$ are clearly $C$-stable. Since $([\sigma]-[1])^{|P|}=\left[\sigma^{|P|}\right]-[1]=0$ for all $\sigma \in P$, we have $I^{M}=0$ for $M$ sufficiently big. Furthermore, the group $P$ acts trivially on the successive quotients $I^{r} / I^{r+1}, r \geq 0$. Thus we have a finite filtration

$$
k[H]=k[P] * C=I^{0} * C \supseteq I^{1} * C \supseteq I^{2} * C \supseteq \cdots \supseteq I^{M} * C=0
$$


of the regular representation $k[H]$ by $k[H]$-submodules such that the successive quotients split into a direct sum of $k[H]$-modules of the form $k[C]=k[H / P]$. (Here, $I^{r} * C$ denotes the ideal $\oplus_{\sigma \in C} I^{r}[\sigma]$ in the twisted group ring $\oplus_{\sigma \in C} k[P][\sigma]=$ $k[P] * C=k[H]$.) Thus the class $[k[H]]$ is a sum of copies of the class $[\mathrm{k}[\mathrm{C}]]$, and Lemma 3.4 follows by a dimension count.

Lemma 3.5. Let $P \in X$. Then we have:

$$
\left[k\left[G_{P}\right]\right]=e_{P}^{\mathrm{w}} \cdot\left[k\left[G_{P} / G_{P, 1}\right]\right]=e_{P}^{\mathrm{w}} \cdot \sum_{d=0}^{e_{P}^{\mathrm{t}}-1}\left[\chi_{P}^{d}\right] \quad \text { in } \quad K_{0}\left(G_{P}, k\right)
$$

Proof. We have seen in the proof of Theorem 3.1 that the character $\chi_{P}$ : $G_{P} \rightarrow k^{\times}$induces a primitive character of the cyclic group $G_{P} / G_{P, 1}$ of order $e_{P}^{\mathrm{t}}$. Hence, Lemma 3.5 follows from Lemma 3.4.

Theorem 3.1 implies the following global relation in $K_{0}(G, k)$ between the representations $\operatorname{Ind}_{G_{P}}^{G}\left(\chi_{P}^{d}\right), P \in X, d=0, \ldots, e_{P}^{\mathrm{t}}-1$.

Corollary 3.6. For $P \in X$ and $d \in\left\{0, \ldots, e_{P}^{\mathrm{t}}-1\right\}$ we put

$$
n_{P, d}:=e_{P}^{\mathrm{w}}\left(d+\frac{\left(e_{P}^{\mathrm{w}}-1\right)\left(e_{P}^{\mathrm{t}}+1\right)+\sum_{s \geq 2}\left(\left|G_{P, s}\right|-1\right)}{2}\right) \in \mathbb{N} .
$$

Then the element

$$
\sum_{P \in X} \sum_{d=0}^{e_{P}^{\mathrm{t}}-1} n_{P, d} \cdot\left[\operatorname{Ind}_{G_{P}}^{G}\left(\chi_{P}^{d}\right)\right] \in K_{0}(G, k)
$$

is divisible by $n=|G|$ in $K_{0}(G, k)$.

Proof. Applying Theorem 3.1 to $\mathcal{E}=\mathcal{O}_{X}$ we obtain

$$
\begin{aligned}
& \chi\left(G, X, \mathcal{O}_{X}\right) \\
& =\left(\left(1-g_{Y}\right)-\frac{1}{2 n} \sum_{P \in X}\left(\left(e_{P}^{\mathrm{w}}-1\right)\left(e_{P}^{\mathrm{t}}+1\right)+\sum_{s \geq 2}\left(\left|G_{P, s}\right|-1\right)\right)\right) \cdot[k[G]] \\
& \quad-\frac{1}{n} \sum_{P \in X} \sum_{d=1}^{e_{P}^{\mathrm{t}}-1} e_{P}^{\mathrm{w}} d\left[\operatorname{Ind}_{G_{P}}^{G}\left(\chi_{P}^{d}\right)\right]
\end{aligned}
$$

in $K_{0}(G, k)_{\mathbb{Q}}$. Now using Lemma 3.5 we obtain Corollary 3.6. 
4. Galois structure in the weakly ramified case. In this section we will generalize several results of Kani and Nakajima on the Galois module structure of Zariski cohomology groups of curves from the tamely ramified to the weakly ramified case.

We keep the assumptions and notations introduced in $\S 2$ and $\S 3$. In addition we assume in this section that the cover $\pi: X \rightarrow Y$ is weakly ramified.

We begin with recalling the following crucial properties of weakly ramified covers.

LEMMA 4.1. For any $P \in X$, the first ramification group $G_{P, 1}$ is an abelian group of exponent $p$, the factor group $G_{P} / G_{P, 1}$ is cyclic of order prime to $p$ and the natural action of $G_{P} / G_{P, 1}$ on $G_{P, 1} \backslash\{1\}$ is free. In particular, $G_{P}$ is the semidirect product of $G_{P, 1}$ and $G_{P} / G_{P, 1}$ and we have: $e_{P}^{\mathrm{W}} \equiv 1 \bmod e_{P}^{\mathrm{t}}$.

Proof. This follows from the pages 74-77 in $\S 2$, Ch. IV of Serre's book [Se1], see in particular Proposition 9 and the corollaries of Proposition 7.

LEMMA 4.2. Let $H$ be the semi-direct product of a finite $p$-group $P$ with a finite group $C$ which acts on $P$. We assume that the action of $C$ on $P \backslash\{1\}$ is free, and we put a $:=(|P|-1) /|C| \in \mathbb{N}$. Furthermore let $V$ be a $k$-representation of $C$ (of finite dimension) which we also view as a $k$-representation of $H$ via the canonical projection $H \rightarrow C$. Let $\operatorname{Cov}(V)$ denote the $k[H]$-projective cover of $V$. Then we have:

$$
[\operatorname{Cov}(V)]=[V]+a \cdot \operatorname{dim}_{k}(V) \cdot[k[C]] \quad \text { in } \quad K_{0}(H, k)
$$

Proof. The order of $C$ is prime to $p$ by assumption. Hence every $k[C]$-module, in particular the $k[C]$-module $V$, is projective. Thus the induced representation $\operatorname{Ind}_{C}^{H}(V)$ is a projective $k[H]$-module. Furthermore we have an obvious $k[H]$ epimorphism $\operatorname{Ind}_{C}^{H}(V) \rightarrow V$. Since $V \cong \operatorname{Ind}_{C}^{H}(V) / \operatorname{rad}(k[H]) \operatorname{Ind}_{C}^{H}(V)$, the $k[H]$ module $\operatorname{Ind}_{C}^{H}(V)$ is minimal with these properties, i.e., $\operatorname{Cov}(V) \cong \operatorname{Ind}_{C}^{H}(V)$. Furthermore, for all $x \in P$ and $\xi, \eta \in C$ we obviously have:

$$
(x, \xi) \cdot(1, \eta) \cdot(x, \xi)^{-1}=\left(x \cdot\left(\xi \eta \xi^{-1}\right)\left(x^{-1}\right), \xi \eta \xi^{-1}\right) \quad \text { in } \quad P \rtimes C=H .
$$

Hence, by assumption on the action of $C$ on $P$, the intersection $C \cap \sigma C \sigma^{-1}$ is trivial for all $\sigma \in H \backslash C$. Using Mackey's double coset theorem (see Theorem 44.2 on p. 324 in [CR1]), we thus obtain:

$$
\begin{aligned}
\operatorname{Res}_{C}^{H}\left(\operatorname{Ind}_{C}^{H}(V)\right) & \cong \bigoplus_{\sigma \in C \backslash G / C} \operatorname{Ind}_{C \cap \sigma C \sigma^{-1}}^{C}\left(\operatorname{Res}_{C \cap \sigma C \sigma^{-1}}^{C}\left(\left(\sigma^{-1}\right)^{*}(V)\right)\right) \\
& =V \oplus\left(\bigoplus^{a \cdot \operatorname{dim}_{k}(V)} k[C]\right) .
\end{aligned}
$$


Finally, the restriction homomorphism $\operatorname{Res}_{C}^{H}: K_{0}(H, k) \rightarrow K_{0}(C, k)$ is bijective since the irreducible $k$-representations of $C$ considered as $k$-representations of $H$ are the only irreducible $k$-representations of $H$ (because $k[H] / \operatorname{rad}(k[H]) \cong k[C]$ ). This proves Lemma 4.2.

In the sequel, the $k\left[G_{P}\right]$-projective cover of the $k\left[G_{P}\right]$-module $\chi_{P}^{d}$ is denoted by $\operatorname{Cov}\left(\chi_{P}^{d}\right)$ (for all $P \in X$ and $d \in \mathbb{Z}$ ). By Lemma 4.2, we have $\left[\operatorname{Cov}\left(\chi_{P}^{d}\right)\right]=$ $\left[\chi_{P}^{d}\right]+\frac{e_{P}^{\mathrm{w}}-1}{e_{P}^{\mathrm{t}}}\left[k\left[G_{P} / G_{P, 1}\right]\right]$ in $K_{0}\left(G_{P}, k\right)$. The following theorem gives a global relation between the projective $k[G]$-modules $\operatorname{Ind}_{G_{P}}^{G}\left(\operatorname{Cov}\left(\chi_{P}^{d}\right)\right), P \in X, d=$ $1, \ldots, e_{P}^{\mathrm{t}}-1$. On the one hand, this relation should be considered as a necessary condition for an arbitrary family of characters $\rho_{P}: G_{P} \rightarrow k^{\times}, P \in X$, to arise as the family of characters $\chi_{P}, P \in X$, associated with a weakly ramified $G$-cover $\pi$. On the other hand, it defines the important ramification module $N_{G, X}$ (used in the next theorem) depending only on the action of $G$ on $X$. Note that $N_{G, X}$ is determined by purely local data, but needs a global divisibility argument for its definition. In particular, the divisibility argument does not hold fibre by fibre in general; in other words, Theorem 4.3 would not be true, if we would omit the direct sum over $P \in X$ on the right-hand side.

THEOREM 4.3. There is a (unique) projective $k[G]$-module $N_{G, X}$ such that

$$
\bigoplus^{n} N_{G, X} \cong \bigoplus_{P \in X} \bigoplus_{d=1}^{e_{P}^{\mathrm{t}}-1} \bigoplus^{e_{P}^{\mathrm{w}} \cdot d} \operatorname{Ind}_{G_{P}}^{G}\left(\operatorname{Cov}\left(\chi_{P}^{d}\right)\right)
$$

The class of $N_{G, X}$ in $K_{0}(k[G])$ is given by

$$
\left[N_{G, X}\right]=\left(1-g_{Y}\right) \cdot[k[G]]-\chi\left(G, X, \mathcal{O}_{X}(E)\right)
$$

where $E$ denotes the G-equivariant divisor $E:=\sum_{P \in X}\left(e_{P}^{\mathrm{w}}-1\right)[P]$.

Remark 4.4. If $\pi$ is assumed to be not only weakly but tamely ramified, then the projective $k[G]$-module on the right-hand side has the following somewhat simpler shape:

$$
\bigoplus_{P \in X} \bigoplus_{d=1}^{e_{P}-1} \bigoplus^{d} \operatorname{Ind}_{G_{P}}^{G}\left(\chi_{P}^{d}\right)
$$

In particular, Theorem 4.3 generalizes the first part of Theorem 2 in Kani's paper [Ka] and Theorem 2(i) in Nakajima's paper [Na2]. 
Proof of Theorem 4.3. We have in $K_{0}(G, k)_{\mathbb{Q}}$ :

$$
\begin{aligned}
\chi(G, & \left.X, \mathcal{O}_{X}(E)\right) \\
= & \left(1-g_{Y}\right)[k[G]] \\
& +\frac{1}{n} \sum_{P \in X}\left(\left(e_{P}^{\mathrm{w}}-1\right)-\frac{\left(e_{P}^{\mathrm{w}}-1\right)\left(e_{P}^{\mathrm{t}}+1\right)}{2}\right)[k[G]] \\
& -\frac{1}{n} \sum_{P \in X} e_{P}^{\mathrm{w}} \sum_{d=1}^{e_{P}^{\mathrm{t}}-1} d \cdot\left[\operatorname{Ind}_{G_{P}}^{G}\left(\chi_{P}^{d+1-e_{P}^{\mathrm{w}}}\right)\right] \\
= & \left(1-g_{Y}\right)[k[G]] \\
& -\frac{1}{n} \sum_{P \in X} \frac{\left(e_{P}^{\mathrm{t}}-1\right)\left(e_{P}^{\mathrm{W}}-1\right)}{2}[k[G]] \quad \text { (by Theorem 3.1) } \\
& -\frac{1}{n} \sum_{P \in X} e_{P}^{\mathrm{w}} \sum_{d=0}^{e_{P}^{\mathrm{t}}-1} d \cdot\left[\operatorname{Ind}_{G_{P}}^{G}\left(\chi_{P}^{d}\right)\right] \quad\left(\operatorname{since} e_{P}^{\mathrm{w}} \equiv 1 \bmod e_{P}^{\mathrm{t}}\right. \text { by Lemma 4.1) } \\
= & \left(1-g_{Y}\right)[k[G]] \\
& -\frac{1}{n} \sum_{P \in X} \frac{\left(e_{P}^{\mathrm{t}}-1\right) e_{P}^{\mathrm{t}}}{2} \cdot \frac{e_{P}^{\mathrm{w}}-1}{e_{P}^{\mathrm{t}}}[k[G]] \\
& -\frac{1}{n} \sum_{P \in X} e_{P}^{\mathrm{w}} \sum_{d=0}^{e_{P}^{\mathrm{t}}-1} d \cdot\left(\left[\operatorname{Ind}_{G_{P}}^{G}\left(\operatorname{Cov}\left(\chi_{P}^{d}\right)\right)\right]-\frac{e_{P}^{\mathrm{w}}-1}{e_{P}^{\mathrm{t}}}\left[\operatorname{Ind}_{G_{P}}^{G}\left(k\left[G_{P} / G_{P, 1}\right]\right)\right]\right)
\end{aligned}
$$

(by Lemma 4.1 and Lemma 4.2)

$$
\begin{aligned}
= & \left(1-g_{Y}\right)[k[G]] \\
& -\frac{1}{n} \sum_{P \in X} e_{P}^{\mathrm{w}} \sum_{d=0}^{e_{P}^{\mathrm{t}}-1} d \cdot\left[\operatorname{Ind}_{G_{P}}^{G}\left(\operatorname{Cov}\left(\chi_{P}^{d}\right)\right)\right]
\end{aligned}
$$

Hence, by Theorem 2.1(a), the class of the projective $k[G]$-module

$$
\bigoplus_{P \in X} \bigoplus_{d=1}^{e_{P}^{\mathrm{t}}-1} \bigoplus^{d \cdot e_{P}^{\mathrm{w}}} \operatorname{Ind}_{G_{P}}^{G}\left(\operatorname{Cov}\left(\chi_{P}^{d}\right)\right)
$$

in $K_{0}(k[G])$ is divisible by $n$ in $K_{0}(k[G])$. Writing the elements of $K_{0}(k[G])$ as integral linear combinations of a basis of $K_{0}(k[G])$ consisting of indecomposable projective $k[G]$-modules, we see that the quotient is again the class of a projective $k[G]$-module, say $N_{G, X}$. Since two projective $k[G]$-modules whose classes in $K_{0}(k[G])$ are equal are already isomorphic, Theorem 4.3 is now proved.

The following theorem expresses the equivariant Euler characteristic $\chi(G, X$, $\left.\mathcal{O}_{X}(D)\right)$ as an integral linear combination of classes of explicit projective $k[G]$ modules (for any $G$-equivariant divisor $D$ as in Theorem 2.1(a)). 
Theorem 4.5. Let $D=\sum_{P \in X} n_{P}[P]$ be a G-equivariant divisor on $X$ with $n_{P} \equiv-1 \bmod e_{P}^{\mathrm{W}}$ for all $P \in X$. For any $P \in X$, we write

$$
n_{P}=\left(e_{P}^{\mathrm{w}}-1\right)+\left(l_{P}+m_{P} e_{P}^{\mathrm{t}}\right) e_{P}^{\mathrm{W}}
$$

with $l_{P} \in\left\{0, \ldots, e_{P}^{\mathrm{t}}-1\right\}$ and $m_{P} \in \mathbb{Z}$. Furthermore, for any $Q \in Y$, we choose a preimage $\tilde{Q} \in X$ of $Q$ under $\pi$. Then we have in $K_{0}(k[G])$ :

$$
\begin{aligned}
& \chi\left(G, X, \mathcal{O}_{X}(D)\right) \\
& \quad=-\left[N_{G, X}\right]+\sum_{Q \in Y} \sum_{d=1}^{l_{\tilde{Q}}}\left[\operatorname{Ind}_{G_{\tilde{Q}}}^{G}\left(\operatorname{Cov}\left(\chi_{\tilde{Q}}^{-d}\right)\right)\right]+\left(1-g_{Y}+\sum_{Q \in Y} m_{\tilde{Q}}\right)[k[G]] .
\end{aligned}
$$

Remark 4.6. Note that $l_{P}=0=m_{P}$ for all but finitely many $P \in X$. If $\pi$ is tamely ramified at $P$, then $l_{P}$ is obviously the unique number in $\left\{0, \ldots, e_{P}^{\mathrm{t}}-\right.$ 1) such that $\mathcal{O}_{X}(D)(P) \cong \chi_{P}^{-l_{P}}$ (as $k\left[G_{P}\right]$-modules). If $\pi$ is tamely ramified everywhere, then Theorem 4.5 implies the congruence

$$
\chi\left(G, X, \mathcal{O}_{X}(D)\right) \equiv-\left[N_{G, X}\right]+\sum_{Q \in Y} \sum_{d=1}^{l_{\tilde{Q}}}\left[\operatorname{Ind}_{G}^{G}\left(\chi_{\tilde{Q}}^{-d}\right)\right] \bmod \mathbb{Z}[k[G]]
$$

for an arbitrary $G$-equivariant divisor $D$ on $X$. This congruence is a reformulation of Theorem 2(ii) in [Na2] applied to $\mathcal{E}:=\mathcal{O}_{X}(D)$.

Proof of Theorem 4.5. Let $E$ denote the divisor $\sum_{P \in X}\left(e_{P}^{\mathrm{W}}-1\right)[P]$ as in Theorem 4.3. We first compute the difference $\chi\left(G, X, \mathcal{O}_{X}(D)\right)-\chi\left(G, X, \mathcal{O}_{X}(E)\right)$ in $K_{0}(G, k)_{\mathbb{Q}}$ :

$$
\begin{aligned}
& \chi\left(G, X, \mathcal{O}_{X}(D)\right)-\chi\left(G, X, \mathcal{O}_{X}(E)\right) \\
&= \frac{1}{n} \sum_{P \in X} n_{P}[k[G]]-\frac{1}{n} \sum_{P \in X}\left(e_{P}^{\mathrm{w}}-1\right)[k[G]] \\
& \quad-\frac{1}{n} \sum_{P \in X} e_{P}^{\mathrm{w}} \sum_{d=1}^{e_{P}^{\mathrm{t}}-1} d\left[\operatorname{Ind}_{G_{P}}^{G}\left(\chi_{P}^{d-n_{P}}\right)\right]+\frac{1}{n} \sum_{P \in X} e_{P}^{\mathrm{w}} \sum_{d=1}^{e_{P}^{\mathrm{t}}-1} d\left[\operatorname{Ind}_{G_{P}}^{G}\left(\chi_{P}^{d+1-e_{P}^{\mathrm{w}}}\right)\right]
\end{aligned}
$$

(by Theorem 3.1)

$$
=\frac{1}{n} \sum_{P \in X}\left(l_{P}+m_{P} e_{P}^{\mathrm{t}}\right) e_{P}^{\mathrm{w}}[k[G]]
$$$$
-\frac{1}{n} \sum_{P \in X} e_{P}^{\mathrm{w}}\left(\sum_{d=0}^{e_{P}^{\mathrm{t}}-1} d\left[\operatorname{Ind}_{G_{P}}^{G}\left(\chi_{P}^{d-l_{P}}\right)\right]-\sum_{d=0}^{e_{P}^{\mathrm{t}}-1} d\left[\operatorname{Ind}_{G_{P}}^{G}\left(\chi_{P}^{d}\right)\right]\right)
$$

(since $e_{P}^{\mathrm{W}} \equiv 1 \bmod e_{P}^{\mathrm{t}}$ by Lemma 4.1) 


$$
\begin{aligned}
= & \frac{1}{n} \sum_{P \in X} m_{P} e_{P}[k[G]]+\frac{1}{n} \sum_{P \in X} l_{P}\left(e_{P}^{\mathrm{w}}-1\right)[k[G]]+\frac{1}{n} \sum_{P \in X} l_{P}[k[G]] \\
& -\frac{1}{n} \sum_{P \in X} e_{P}^{\mathrm{w}}\left(\sum_{d=0}^{e_{P}^{\mathrm{t}}-1} l_{P}\left[\operatorname{Ind}_{G_{P}}^{G}\left(\chi_{P}^{d}\right)\right]-\sum_{d=e_{P}^{\mathrm{t}}-l_{P}}^{e_{P}^{\mathrm{t}}-1} e_{P}^{\mathrm{t}}\left[\operatorname{Ind}_{G_{P}}^{G}\left(\chi_{P}^{d}\right)\right]\right) \\
= & \frac{1}{n} \sum_{P \in X} m_{P} e_{P}[k[G]]+\frac{1}{n} \sum_{P \in X} l_{P}\left(e_{P}^{\mathrm{w}}-1\right) e_{P}^{\mathrm{w}}\left[\operatorname{Ind}_{G_{P}}^{G}\left(k\left[G_{P} / G_{P, 1}\right]\right)\right] \\
& +\frac{1}{n} \sum_{P \in X} e_{P}^{\mathrm{w}} e_{P}^{\mathrm{t}} \sum_{d=e_{P}^{\mathrm{t}}-l_{P}}^{e_{P}^{\mathrm{t}}-1}\left[\operatorname{Ind}_{G_{P}}^{G}\left(\chi_{P}^{d}\right)\right] \\
= & \sum_{P \in X} \frac{e_{P}}{n} m_{P}[k[G]] \\
& +\sum_{P \in X} \frac{e_{P}}{n} \sum_{d=e_{P}^{\mathrm{t}}-l_{P}}^{e_{P}^{\mathrm{t}}-1}\left(\frac{e_{P}^{\mathrm{w}}-1}{e_{P}^{\mathrm{t}}}\left[\operatorname{Ind}_{G_{P}}^{G}\left(k\left[G_{P} / G_{P, 1}\right]\right)\right]+\left[\operatorname{Ind}_{G_{P}}^{G}\left(\chi_{P}^{d}\right)\right]\right) \\
= & \sum_{Q \in Y} m_{\tilde{Q}}[k[G]]+\sum_{Q \in Y} \sum_{d=1}^{l_{\tilde{Q}}}\left[\operatorname{Ind}_{G_{\tilde{Q}}}^{G}\left(\operatorname{Cov}\left(\chi_{\tilde{Q}}^{-d}\right)\right)\right] \quad\left(\text { since } e_{P}=e_{P}^{\mathrm{w}} e_{P}^{\mathrm{t}}\right)
\end{aligned}
$$

(by Lemma 4.1 and Lemma 4.2).

This result for the difference $\chi\left(G, X, \mathcal{O}_{X}(D)\right)-\chi(G, X, \mathcal{O}(E))$ together with the formula

$$
\chi\left(G, X, \mathcal{O}_{X}(E)\right)=\left(1-g_{Y}\right)[k[G]]-\left[N_{G, X}\right]
$$

(see Theorem 4.3) now obviously implies the desired formula in Theorem 4.5.

The ideal sheaf of the reduced effective divisor on $X$ consisting of all (wildly) ramified points plays a central role in Pink's paper [Pi]. The following corollary computes the Galois module structure of its first cohomology group.

COROLlaRY 4.7. Let $S$ be a $G$-stable non-empty finite set of closed points on $X$ which contains all wildly ramified points, i.e. all points $P \in X$ with $e_{P}^{\mathrm{w}} \neq 1$. Let $\mathcal{I}(S)$ denote the ideal sheaf of $S$. Then the $k[G]$-module $H^{1}(X, \mathcal{I}(S))$ is stably isomorphic to $N_{G, X} \oplus\left(\oplus_{Q \in \pi(S)} \operatorname{Ind}_{G_{\tilde{Q}}}^{G}\left(\operatorname{Cov}\left(\chi_{\tilde{Q}}^{0}\right)\right)\right)$. More precisely we have:

$$
H^{1}(X, \mathcal{I}(S)) \oplus k[G] \cong N_{G, X} \oplus\left(\bigoplus_{Q \in \pi(S)} \operatorname{Ind}_{G_{\tilde{Q}}}^{G}\left(\operatorname{Cov}\left(\chi_{\tilde{Q}}^{0}\right)\right)\right) \oplus\left(\bigoplus^{g_{Y}} k[G]\right)
$$


Proof. Since $S \neq \emptyset$, we have $H^{0}(X, \mathcal{I}(S))=0$. Hence we obtain the following equality in $K_{0}(G, k)$ :

$$
\begin{aligned}
& {\left[H^{1}(X, \mathcal{I}(S))\right]=-\chi\left(G, X, \mathcal{O}_{X}\left(\sum_{P \in S}-[P]\right)\right)} \\
& =\left[N_{G, X}\right]-\sum_{Q \in \pi(S)} \sum_{d=1}^{e_{\tilde{Q}}^{\mathrm{t}}-1}\left[\operatorname{Ind}_{G_{\tilde{Q}}}^{G}\left(\operatorname{Cov}\left(\chi_{\tilde{Q}}^{-d}\right)\right)\right]+\sum_{Q \in \pi(S)}[k[G]]+\left(g_{Y}-1\right)[k[G]] \\
& =\left[N_{G, X}\right]+\sum_{Q \in \pi(S)}\left[\operatorname{Ind}_{G_{\tilde{Q}}}^{G}\left(\operatorname{Cov}\left(\chi_{\tilde{Q}}^{0}\right)\right)\right]+\left(g_{Y}-1\right)[k[G]] \quad \text { (by Theorem 4.5) }
\end{aligned}
$$

(by Lemma 4.1 and Lemma 4.2).

Furthermore, $H^{1}(X, \mathcal{I}(S))$ is a projective $k[G]$-module by Theorem 2.1(a). Since two projective $k[G]$-modules whose classes are equal in $K_{0}(G, k)$ are already isomorphic, Corollary 4.7 is now proved.

The following corollary computes the Galois module structure of the space of global meromorphic differentials on $X$ which are logarithmic along all ramified points. It generalizes the second part of Theorem 2 in Kani's paper [Ka] from the tamely ramified to the weakly ramified case.

Corollary 4.8. Let $S$ be a G-stable non-empty finite set of closed points on $X$ which contains all ramified points. Let $\Omega_{X}(S)$ denote the sheaf of meromorphic differentials on $X$ which are logarithmic along $S$. Then the $k[G]$-module $H^{0}\left(X, \Omega_{X}(S)\right) \oplus N_{G, X}$ is free of rank $|S / G|+g_{Y}-1$.

Proof. We use the notations introduced in the proof of Corollary 2.3. Then the divisor $D=K_{X}+\sum_{P \in S}[P]$ satisfies the condition of Theorem 4.5 and the corresponding integers $l_{P}, m_{P}, P \in X$, are given as follows: $l_{P}=0$ for all $P \in X$, $m_{P}=r_{\pi(P)}+1$ for $P \in S$ and $m_{P}=r_{\pi(P)}$ for $P \in X \backslash S$. Thus we obtain:

$$
\begin{aligned}
& {\left[H^{0}\left(X, \Omega_{X}(S)\right) \oplus N_{G, X}\right]=\left[H^{0}\left(X, \mathcal{O}_{X}(D)\right)\right]+\left[N_{G, X}\right]} \\
& =\left(1-g_{Y}+\sum_{Q \in Y} r_{Q}+|S / G|\right)[k[G]] \quad \text { (by Theorem 4.5) } \\
& =\left(g_{Y}-1+|S / G|\right)[k[G]] \quad \text { in } \quad K_{0}(k[G]) \quad\left(\text { since } \operatorname{deg}\left(\Omega_{Y}\right)=2 g_{Y}-2\right) .
\end{aligned}
$$

As in Corollary 4.7, this implies Corollary 4.8.

Remark 4.9. Alternatively, Corollary 4.8 can be derived from Corollary 4.7 using the Serre duality isomorphism $H^{0}\left(X, \Omega_{X}(S)\right) \cong H^{1}(X, \mathcal{I}(S))^{*}$ and the iso- 
morphism

$$
N_{G, X}^{*} \oplus\left(\bigoplus_{Q \in \pi(S)} \operatorname{Ind}_{G}^{G}\left(\operatorname{Cov}\left(\chi_{\tilde{Q}}^{0}\right)\right)^{*}\right) \oplus N_{G, X} \cong \bigoplus^{|S / G|} k[G]
$$

which may easily be checked.

School of MATHEMATICS, University of SOUTHAMPTON, SOUTHAMPTON SO17 1BJ, UNITED KINGDOM

E-mail: bk@maths.soton.ac.uk

\section{REFERENCES}

[BFQ] P. Baum, W. Fulton, and G. Quart, Lefschetz-Riemann-Roch for singular varieties, Acta Math. 143 (1979), 193-211.

[Bo1] N. Borne, Une formule de Riemann-Roch équivariante pour les courbes, Thèse, Université Bordeaux I, 2000.

[Bo2] Structure du groupe de Grothendieck équivariant d'une courbe et modules galoisiens, Bull. Soc. Math. France 130 (2002), 101-121.

[Ch] T. Chinburg, Galois structure of de Rham cohomology of tame covers of schemes, Ann. of Math. 139 (1994), 443-490.

[CR1] C. W. Curtis and I. Reiner, Representation Theory of Finite Groups and Associative Algebras, Pure Appl. Math., XI, Interscience Publishers, John Wiley \& Sons, New York, 1962.

[CR2] Methods of representation theory, with applications to finite groups and orders, vol. I, Pure Appl. Math., Interscience Publishers, John Wiley \& Sons, New York, 1981.

[EL] G. Ellingsrud and K. Lønsted, An equivariant Lefschetz formula for finite reductive groups, Math. Ann. 251 (1980), 253-261.

[Er1] B. Erez, The Galois structure of the square root of the inverse different, Math. Z. 208 (1991), 239-255.

[Er2] Geometric trends in Galois module theory, Galois Representations in Arithmetic Algebraic Geometry (Durham, 1996), London Math. Soc. Lecture Note Ser., vol. 254, Cambridge Univ. Press, Cambridge, 1998, 115-145.

[FL] W. Fulton and S. Lang, Riemann-Roch Algebra, Grundlehren Math. Wiss., vol. 277, Springer-Verlag, New York, 1985.

[Ha] R. Hartshorne, Algebraic Geometry, Grad. Texts in Math., vol. 52, Springer-Verlag, New York, 1977.

[Has] H. Hasse, Theorie der relativ-zyklischen algebraischen Funktionenkörper, insbesondere bei endlichem Konstantenkörper, J. Reine Angew. Math. 172 (1934), 37-54 (= Mathematische Abhandlungen, band 2, de Gruyter, Berlin, 1975, 133-150).

[Ka] E. Kani, The Galois-module structure of the space of holomorphic differentials of a curve, J. Reine Angew. Math. 367 (1986), 187-206.

[Kö1] B. Köck, The Lefschetz theorem in higher equivariant $K$-theory, Comm. Algebra 19 (1991), 34113422.

[Kö2] Computing the equivariant Euler characteristic of Zariski and étale sheaves on curves, preprint, 2001

[Na1] S. Nakajima, On Galois module structure of the cohomology groups of an algebraic variety, Invent. Math. 75 (1984), 1-8.

[Na2] Galois module structure of cohomology groups for tamely ramified coverings of algebraic varieties, J. Number Theory 22 (1986), 115-123. 
[Na3] Action of an automorphism of order $p$ on cohomology groups of an algebraic curve, $J$. Pure Appl. Algebra 42 (1986), 85-94.

[Ne] J. Neukirch, Algebraische Zahlentheorie, Springer-Verlag, Berlin, 1992.

[No] E. Noether, Normalbasis bei Körpern ohne höhere Verzweigung, J. Reine Angew. Math. 167 (1931), 147-152.

[Pi] R. Pink, Euler-Poincaré formula in equal characteristic under ordinariness assumptions, Manuscripta Math. 102 (2000), 1-24.

[Se1] J.-P. Serre, Corps locaux, Publications de l'Institut de Mathématique de l'Université de Nancago, vol. VIII, Hermann, Paris, 1962.

[Se2] L Linear Representations of Finite Groups, Grad. Texts in Math., vol. 42, Springer-Verlag, New York, 1977.

[Sw] R. G. Swan, Induced representations and projective modules, Ann. of Math. (2) 71 (1960), 552-578.

[U11] S. Ullom, Galois cohomology of ambiguous ideals, J. Number Theory 1 (1969), 11-15.

[U12] Normal bases in Galois extensions of number fields, Nagoya Math. J. 34 (1969), 153167.

[U13] $\longrightarrow$ Integral normal bases in Galois extensions of local fields, Nagoya Math. J. 39 (1970), $141-148$.

[Vi] S. Vinatier, Structure galoisienne dans les extensions faiblement ramifiees de $\mathbb{Q}$, J. Number Theory 91 (2001), 126-152. 